\title{
Arithmetic of the modular function $j_{1,4}$
}

\author{
by
}

\author{
Chang Heon Kim and Ja Kyung Koo (Taejon)
}

We find a generator $j_{1,4}$ of the function field on the modular curve $X_{1}(4)$ by means of classical theta functions $\theta_{2}$ and $\theta_{3}$, and estimate the normalized generator $N\left(j_{1,4}\right)$ which becomes the Thompson series of type $4 C$. With these modular functions we investigate some number theoretic properties.

1. Introduction. Let $\mathfrak{H}$ be the complex upper half plane and let $\Gamma_{1}(N)$ be a congruence subgroup of $S L_{2}(\mathbb{Z})$ whose elements are congruent to $\left(\begin{array}{ll}1 & * \\ 0 & 1\end{array}\right)$ $\bmod N(N=1,2, \ldots)$. Since the group $\Gamma_{1}(N)$ acts on $\mathfrak{H}$ by linear fractional transformations, we get the modular curve $X_{1}(N)=\Gamma_{1}(N) \backslash \mathfrak{H}^{*}$, as the projective closure of the smooth affine curve $\Gamma_{1}(N) \backslash \mathfrak{H}$, with genus $g_{1, N}$. Since $g_{1, N}=0$ only for the eleven cases $1 \leq N \leq 10$ and $N=12([9])$, the function field $K\left(X_{1}(4)\right)$ of the curve $X_{1}(4)$ is a rational function field over $\mathbb{C}$.

In this article we will first find the field generator $j_{1,4}$ in Section 3 by making use of the classical Jacobi theta functions $\theta_{2}$ and $\theta_{3}$. Furthermore, we will show that $\mathbb{Q}\left(j_{1,4}\right)=\mathbb{Q}(j, j(4 z))\left(j=\right.$ the modular invariant of $\left.S L_{2}(\mathbb{Z})\right)$ is the field of all modular functions in $K\left(X_{1}(4)\right)$ whose Fourier coefficients with respect to $q\left(=e^{2 \pi i z}, z \in \mathfrak{H}\right)$ are rational numbers. We will also find the relation between two modular functions $j_{1,4}$ and $j_{4}=\theta_{3}(z / 2) / \theta_{4}(z / 2)$ ([8]). In Section 4 we will estimate the normalized generator $N\left(j_{1,4}\right)$ of $K\left(X_{1}(4)\right)$ as the type of the field which turns out to be the Thompson series of type $4 C$, and will investigate the replication formulas for the coefficients of $N\left(j_{1,4}\right)$. When $\tau \in \mathfrak{H} \cap \mathbb{Q}(\sqrt{-d})$ for a square-free positive integer $d$, we will show that $N\left(j_{1,4}\right)(\tau)$ becomes an algebraic integer. Finally, in Section 5 we will construct some class fields over an imaginary quadratic field by applying Shimura theory and standard results of complex multiplication to our function $j_{1,4}$.

Throughout the article we adopt the following notations:

1991 Mathematics Subject Classification: 11F03, 11F11, 11F22, 11R04, 11R37, 14H55.

Supported by KOSEF research grant 95-K3-0101 (RCAA). 
- $\mathfrak{H}^{*}$ the extended complex upper half plane,

- $\Gamma(N)=\left\{\gamma \in S L_{2}(\mathbb{Z}) \mid \gamma \equiv I \bmod N\right\}$,

- $\Gamma_{0}(N)$ the Hecke subgroup $\left\{\left(\begin{array}{ll}a & b \\ c & d\end{array}\right) \in \Gamma(1) \mid c \equiv 0 \bmod N\right\}$,

- $\bar{\Gamma}$ the inhomogeneous group of $\Gamma(=\Gamma / \pm I)$,

- $q_{h}=e^{2 \pi i z / h}, z \in \mathfrak{H}$,

- $M_{k}\left(\Gamma_{1}(N)\right)$ the space of modular forms of weight $k$ with respect to the group $\Gamma_{1}(N)$,

- $\mathbb{Z}_{p}$ the ring of $p$-adic integers,

- $\mathbb{Q}_{p}$ the field of $p$-adic numbers.

2. Generators of $\Gamma_{1}(4)$. Let $\Gamma$ be a congruence subgroups of $\Gamma(1)$ $\left(=S L_{2}(\mathbb{Z})\right)$. A subset $\mathbb{F}$ of $\mathfrak{H}^{*}$ is called a fundamental set for the group $\bar{\Gamma}$ if it contains exactly one representative of each class of points of $\mathfrak{H}^{*}$ equivalent under $\bar{\Gamma}$. A set $\mathbb{F}$ is called a fundamental region if $\mathbb{F}$ contains a fundamental set and, for $z \in \mathbb{F}$ and $\gamma z \in \mathbb{F}$ with $\gamma(\neq I) \in \bar{\Gamma}, z$ is a boundary point of $\mathbb{F}$.

Although the following theorem is well known, we present its proof for the sake of completeness.

THEOREM 1. Let $\bar{\Gamma}$ be a congruence subgroup of $\bar{\Gamma}(1)$ of finite index and $\mathbb{F}$ be a fundamental region for $\bar{\Gamma}$. Then the sides of $\mathbb{F}$ can be grouped into pairs $\lambda_{j}, \lambda_{j}^{\prime}(j=1, \ldots, s)$ in such a way that $\lambda_{j} \subseteq \mathbb{F}$ and $\lambda_{j}^{\prime}=\gamma_{j} \lambda_{j}$ where $\gamma_{j} \in \bar{\Gamma}(j=1, \ldots, s)$. $\gamma_{j}$ 's are called boundary substitutions of $\mathbb{F}$. Furthermore, $\bar{\Gamma}$ is generated by the boundary substitutions $\gamma_{1}, \ldots, \gamma_{s}$.

Proof. For the first part, see [16], p. 58. For any $\gamma \in \bar{\Gamma}$, suppose that there exists a sequence of images of $\mathbb{F}$, that is, $\mathbb{F}, S_{1} \mathbb{F}, S_{2} \mathbb{F}, \ldots, S_{n} \mathbb{F}=\gamma \mathbb{F}$ $\left(S_{j} \in \bar{\Gamma}\right)$, each adjacent to its successor. Let $\mathbb{F} \cap S_{1} \mathbb{F} \supseteq \lambda_{j}^{\prime}$. Since $\gamma_{j} \lambda_{j}=$ $\lambda_{j}^{\prime}$ and $\gamma_{j} \mathbb{F}$ is another fundamental region, $\gamma_{j} \mathbb{F}=S_{1} \mathbb{F}$, which yields that $S_{1}$ $=\gamma_{j}$. Then $\gamma_{j} \lambda_{i}, \gamma_{j} \lambda_{i}^{\prime}(i=1, \ldots, s)$ form the sides of $S_{1} \mathbb{F}$ and $\left(\gamma_{j} \gamma_{i} \gamma_{j}^{-1}\right) \gamma_{j} \lambda_{i}$ $=\gamma_{j} \lambda_{i}^{\prime}$, i.e., $\gamma_{j} \gamma_{i} \gamma_{j}^{-1}(i=1, \ldots, s)$ are boundary substitutions of $S_{1} \mathbb{F}$.

Now, we use induction on $n$ to show that $S_{n}(=\gamma)$ is generated by $\gamma_{1}, \ldots, \gamma_{s}$ and boundary substitutions are also generated by them. The case $n=1$ has been done. Denote the sides of $S_{n-1} \mathbb{F}$ by $\mu_{i}, \mu_{i}^{\prime}(i=1, \ldots, s)$. Let $L_{i} \mu_{i}=\mu_{i}^{\prime}$ for $i=1, \ldots, s$. Then, by induction hypothesis, $S_{n-1}$ and $L_{i}(i=1, \ldots, s)$ are generated by $\gamma_{1}, \ldots, \gamma_{s}$. If $S_{n-1} \mathbb{F} \cap S_{n} \mathbb{F} \supseteq \mu_{j}^{\prime}$, then $L_{j} \mu_{j}=\mu_{j}^{\prime}$ implies that $L_{j} S_{n-1} \mathbb{F}=S_{n} \mathbb{F}$, i.e., $S_{n}=L_{j} S_{n-1}$. Hence, it is generated by $\gamma_{1}, \ldots, \gamma_{s}$. Also, the set of all points in $\mathfrak{H}$ belonging to the region $S_{n} \mathbb{F}$ that can be reached by such sequences is open, and so is its complement in $\mathfrak{H}$ which must be empty by connectedness of $\mathfrak{H}$. This completes the proof of the theorem.

Lemma 2. Let $\alpha=\left(\begin{array}{cc}0 & -1 \\ 2 & 0\end{array}\right)$. Then $\pm \alpha \Gamma(2) \alpha^{-1}= \pm \Gamma_{1}(4)$.

Pr o of. Straightforward. 
It is well known ([17], p. 84) that $\Gamma(2)$ has the following fundamental domain:

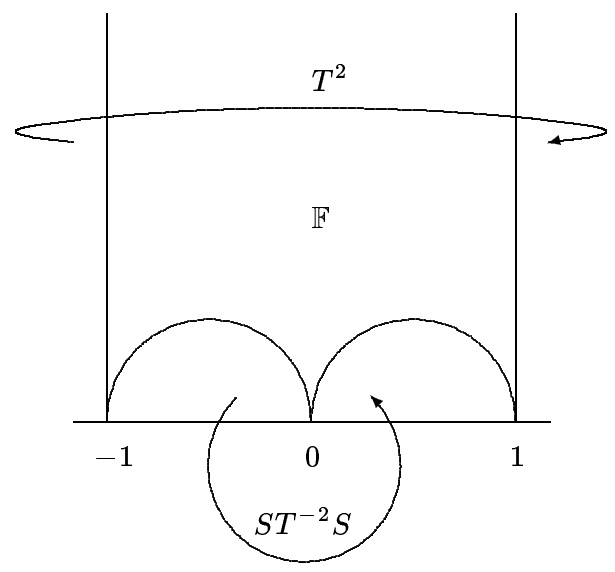

where $T=\left(\begin{array}{ll}1 & 1 \\ 0 & 1\end{array}\right)$ and $S=\left(\begin{array}{cc}0 & -1 \\ 1 & 0\end{array}\right)$. Thus, by Lemma 2 we can come up with the fundamental domain $\alpha \mathbb{F}$ of $\Gamma_{1}(4)$ as follows:

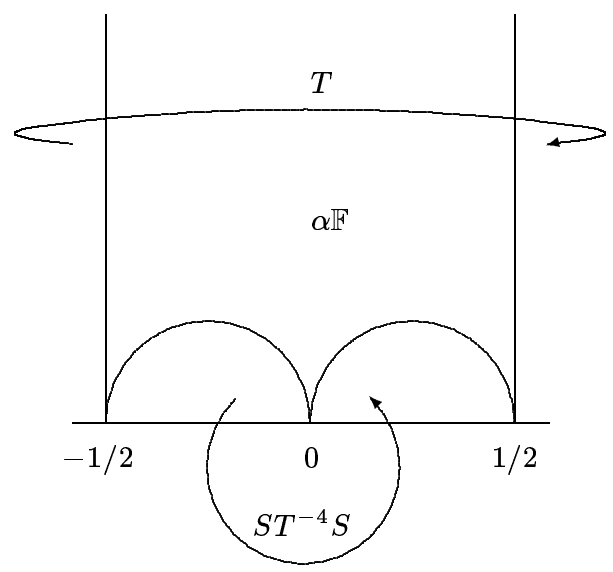

Since $T$ and $S T^{-4} S$ are in $\bar{\Gamma}_{1}(4)$, they generate the group $\bar{\Gamma}_{1}(4)$ by Theorem 1 . There are 3 cusps $\infty, 0, \frac{1}{2}$ in $X_{1}(4)$ as seen in the above figure, whose widths are 1, 4 and 1, respectively. Here we observe that the first two are regular and the last one is irregular.

3. Hauptfunktionen of $K\left(X_{1}(4)\right)$ as a quotient of Jacobi theta functions. First, we recall the Jacobi theta functions $\theta_{2}, \theta_{3}, \theta_{4}$ defined by

$$
\theta_{2}(z)=\sum_{n \in \mathbb{Z}} q_{2}^{(n+1 / 2)^{2}}, \quad \theta_{3}(z)=\sum_{n \in \mathbb{Z}} q_{2}^{n^{2}}, \quad \theta_{4}(z)=\sum_{n \in \mathbb{Z}}(-1)^{n} q_{2}^{n^{2}}
$$

for $z \in \mathfrak{H}$. Then we have the following transformation formulas ([16], pp. 218-219): 


$$
\begin{aligned}
& \theta_{2}(z+1)=e^{\pi i / 4} \theta_{2}(z), \\
& \theta_{3}(z+1)=\theta_{4}(z), \\
& \theta_{4}(z+1)=\theta_{3}(z), \\
& \theta_{2}(-1 / z)=(-i z)^{1 / 2} \theta_{4}(z), \\
& \theta_{3}(-1 / z)=(-i z)^{1 / 2} \theta_{3}(z), \\
& \theta_{4}(-1 / z)=(-i z)^{1 / 2} \theta_{2}(z) .
\end{aligned}
$$

Put $j_{1,4}(z)=\theta_{2}(2 z)^{4} / \theta_{3}(2 z)^{4}$. Then we obtain the following theorem.

TheOREM 3. (i) $\theta_{2}(2 z)^{4}, \theta_{3}(2 z)^{4} \in M_{2}\left(\Gamma_{1}(4)\right)$.

(ii) $K\left(X_{1}(4)\right)=\mathbb{C}\left(j_{1,4}(z)\right)$ and $j_{1,4}(\infty)=0$ (simple zero), $j_{1,4}(0)=1$, $j_{1,4}(1 / 2)=\infty$ (simple pole) .

Proof. For the first part, we must check the invariance of the slash operator and the cusp conditions. Since $T$ and $S T^{-4} S$ generate $\bar{\Gamma}_{1}(4)$, it is enough to check it for these generators.

$$
\begin{aligned}
\left.\theta_{2}(2 z)^{4}\right|_{[T]_{2}} & =\theta_{2}(2 z+2)^{4}=\left(e^{\pi i / 2} \theta_{2}(2 z)\right)^{4} \quad \text { by }(1) \\
& =\theta_{2}(2 z)^{4}, \\
\left.(7) \quad \theta_{2}(2 z)^{4}\right|_{[S]_{2}} & =z^{-2} \theta_{2}(-2 / z)^{4}=z^{-2}\left\{(-i z / 2)^{1 / 2} \theta_{4}(z / 2)\right\}^{4} \quad \text { by }(4) \\
& =-\frac{1}{4} \theta_{4}(z / 2)^{4}, \\
\left.\theta_{2}(2 z)^{4}\right|_{\left[S T^{-4}\right]_{2}} & =-\left.\frac{1}{4} \theta_{4}(z / 2)^{4}\right|_{\left[T^{-4}\right]_{2}}=-\frac{1}{4} \theta_{4}(z / 2)^{4} \quad \text { by }(2) \text { and }(3), \\
\left.\theta_{2}(2 z)^{4}\right|_{\left[S T^{-4} S\right]_{2}} & =-\left.\frac{1}{4} \theta_{4}(z / 2)^{4}\right|_{[S]_{2}}=-\frac{1}{4} z^{-2}\left\{(-2 i z)^{1 / 2} \theta_{2}(2 z)\right\}^{4} \quad \text { by }(6) \\
& =\theta_{2}(2 z)^{4}, \\
\left.\theta_{3}(2 z)^{4}\right|_{[T]_{2}} & =\theta_{3}(2 z+2)^{4}=\theta_{3}(2 z)^{4} \quad \text { by }(2) \text { and }(3), \\
\left.(8) \quad \theta_{3}(2 z)^{4}\right|_{[S]_{2}} & =z^{-2} \theta_{3}(-2 / z)^{4}=z^{-2}\left\{(-i z / 2)^{1 / 2} \theta_{3}(z / 2)\right\}^{4} \quad \text { by }(5) \\
& =-\frac{1}{4} \theta_{3}(z / 2)^{4}, \\
\left.\theta_{3}(2 z)^{4}\right|_{\left[S T^{-4}\right]_{2}} & =-\left.\frac{1}{4} \theta_{3}(z / 2)^{4}\right|_{\left[T^{-4}\right]_{2}} \\
& =-\frac{1}{4} \theta_{3}(z / 2)^{4} \quad \text { by }(2) \text { and }(3), \\
\left.\theta_{3}(2 z)^{4}\right|_{\left[S T^{-4} S\right]_{2}} & =-\left.\frac{1}{4} \theta_{3}(z / 2)^{4}\right|_{[S]_{2}}=-\frac{1}{4} z^{-2}\left\{(-2 i z)^{1 / 2} \theta_{3}(2 z)\right\}^{4} \quad \text { by }(5) \\
& =\theta_{3}(2 z)^{4} .
\end{aligned}
$$

Now we check the boundary conditions.

(i) $s=\infty$ : Since $\theta_{2}(z)=2 q_{8}\left(1+q+q^{3}+\ldots\right)$, we have

$$
\theta_{2}(2 z)^{4}=2^{4} q\left(1+q^{2}+q^{6}+q^{12}+\ldots\right)^{4} .
$$

Hence $\theta_{2}(2 z)^{4}$ has a simple zero at $s=\infty$. On the other hand, $\theta_{3}(2 z)^{4}=$ $\left(\sum_{n \in \mathbb{Z}} q^{n^{2}}\right)^{4}=\left(1+2 q+2 q^{4}+2 q^{9}+\ldots\right)^{4}$. Thus $\left.\theta_{3}(2 z)^{4}\right|_{s=\infty}=1$. 
(ii) $s=0$ :

$$
\begin{aligned}
\left.\theta_{2}(2 z)^{4}\right|_{s=0} & =\left.\lim _{z \rightarrow i \infty} \theta_{2}(2 z)^{4}\right|_{[S]_{2}}=\lim _{z \rightarrow i \infty}-\frac{1}{4} \theta_{4}(z / 2)^{4} \quad \text { by }(7) \\
& =-\frac{1}{4}
\end{aligned}
$$

and

$$
\begin{aligned}
\left.\theta_{3}(2 z)^{4}\right|_{s=0} & =\left.\lim _{z \rightarrow i \infty} \theta_{3}(2 z)^{4}\right|_{[S]_{2}}=\lim _{z \rightarrow i \infty}-\frac{1}{4} \theta_{3}(z / 2)^{4} \quad \text { by }(8) \\
& =-\frac{1}{4} .
\end{aligned}
$$

(iii) $s=1 / 2$ : Observe that $\left(S T^{-2} S\right) \infty=1 / 2$.

Considering the identities

$$
\begin{aligned}
\left.\theta_{2}(2 z)^{4}\right|_{[S]_{2}} & =-\frac{1}{4} \theta_{4}(z / 2)^{4} \quad \text { by }(7), \\
\left.\theta_{2}(2 z)^{4}\right|_{\left[S T^{-2}\right]_{2}} & =-\left.\frac{1}{4} \theta_{4}(z / 2)^{4}\right|_{\left[T^{-2}\right]_{2}}=-\frac{1}{4} \theta_{3}(z / 2)^{4} \quad \text { by }(3), \\
\left.\theta_{2}(2 z)^{4}\right|_{\left[S T^{-2} S\right]_{2}} & =-\left.\frac{1}{4} \theta_{3}(z / 2)^{4}\right|_{[S]_{2}}=-\frac{1}{4} z^{-2}\left\{(-2 i z)^{1 / 2} \theta_{3}(2 z)\right\}^{4} \quad \text { by }(5) \\
& =\theta_{3}(2 z)^{4},
\end{aligned}
$$

we get

$$
\left.\theta_{2}(2 z)^{4}\right|_{s=1 / 2}=\left.\lim _{z \rightarrow i \infty} \theta_{2}(2 z)^{4}\right|_{\left[S T^{-2} S\right]_{2}}=\lim _{z \rightarrow i \infty} \theta_{3}(2 z)^{4}=1 .
$$

The facts that

$$
\begin{aligned}
\left.\theta_{3}(2 z)^{4}\right|_{[S]_{2}} & =-\frac{1}{4} \theta_{3}(z / 2)^{4} \quad \text { by }(8), \\
\left.\theta_{3}(2 z)^{4}\right|_{\left[S T^{-2}\right]_{2}} & =-\left.\frac{1}{4} \theta_{3}(z / 2)^{4}\right|_{\left[T^{-2}\right]_{2}}=-\frac{1}{4} \theta_{4}(z / 2)^{4} \quad \text { by }(2), \\
\left.\theta_{3}(2 z)^{4}\right|_{\left[S T^{-2} S\right]_{2}} & =-\left.\frac{1}{4} \theta_{4}(z / 2)^{4}\right|_{[S]_{2}}=-\frac{1}{4} z^{-2}\left\{(-2 i z)^{1 / 2} \theta_{2}(2 z)\right\}^{4} \quad \text { by }(6) \\
& =\theta_{2}(2 z)^{4}
\end{aligned}
$$

imply

$$
\begin{aligned}
\left.\theta_{3}(2 z)^{4}\right|_{s=1 / 2} & =\left.\lim _{z \rightarrow i \infty} \theta_{3}(2 z)^{4}\right|_{\left[S T^{-2} S\right]_{2}}=\lim _{z \rightarrow i \infty} \theta_{2}(2 z)^{4} \\
& =\lim _{z \rightarrow i \infty} 2^{4} q\left(1+q^{2}+q^{6}+q^{12}+\ldots\right)^{4} \\
& =0 \text { a simple zero. }
\end{aligned}
$$

Now, we prove the second part. From the well-known formula ([19], p. 39) concerning the sum of orders of zeros of modular forms, it follows that $\nu_{0}\left(\theta_{2}(2 z)^{4}\right)=\nu_{0}\left(\theta_{3}(2 z)^{4}\right)=1$. Hence $\theta_{2}(2 z)^{4}$ (resp. $\left.\theta_{3}(2 z)^{4}\right)$ has no other zeros in $X_{1}(4)$ except at $s=\infty$ (resp. $\left.s=1 / 2\right)$. Therefore $\left[K\left(X_{1}(4)\right)\right.$ : $\left.\mathbb{C}\left(j_{1,4}(z)\right)\right]=\nu_{0}\left(j_{1,4}(z)\right)=1$, and so (ii) follows.

Let $K\left(X\left(\Gamma^{\prime}\right)\right)$ be the function field of the modular curve $X\left(\Gamma^{\prime}\right)=\Gamma^{\prime} \backslash \mathfrak{H}^{*}$. Suppose that the genus of $X\left(\Gamma^{\prime}\right)$ is zero. Let $h$ be the width of the cusp $\infty$. By $F$ we mean the field of all modular functions in $K\left(X\left(\Gamma^{\prime}\right)\right)$ whose Fourier coefficients with respect to $q_{h}$ belong to $\mathbb{Q}$. 
Lemma 4. Let $K\left(X\left(\Gamma^{\prime}\right)\right)=\mathbb{C}\left(J^{\prime}\right)$ for some $J^{\prime} \in K\left(X\left(\Gamma^{\prime}\right)\right)$. If $J^{\prime} \in F$, then $F=\mathbb{Q}\left(J^{\prime}\right)$.

P r o of. First, note that $F$ and $\mathbb{C}$ are linearly disjoint over $\mathbb{Q}$. Indeed, let $\mu_{1}, \ldots, \mu_{m}$ be the elements of $\mathbb{C}$ which are linearly independent over $\mathbb{Q}$. Assume that $\sum_{i} \mu_{i} g_{i}=0$ with $g_{i}$ in $F$. Let $g_{i}=\sum_{n} c_{i n} q_{h}^{n}$ with $c_{i n} \in \mathbb{Q}$. Then $\sum_{i} \mu_{i} c_{i n}=0$ for every $n$, so that $c_{i n}=0$ for all $i$ and $n$. Hence $g_{1}=\ldots=g_{m}=0$. We then have the field tower

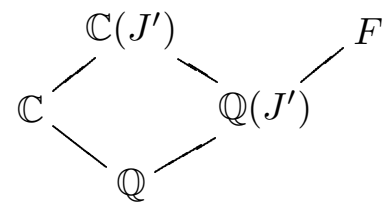

From the tower, we see that $F$ and $\mathbb{C}\left(J^{\prime}\right)$ are linearly disjoint over $\mathbb{Q}\left(J^{\prime}\right)$ by [12], p. 361. Hence,

$$
1 \leq\left[F: \mathbb{Q}\left(J^{\prime}\right)\right] \leq\left[\mathbb{C} F: \mathbb{C}\left(J^{\prime}\right)\right] \leq\left[K\left(X\left(\Gamma^{\prime}\right)\right): K\left(X\left(\Gamma^{\prime}\right)\right)\right]=1,
$$

which yields that $F=\mathbb{Q}\left(J^{\prime}\right)$.

Lemma 5. If $\Gamma^{\prime}=\Gamma_{0}(N)$, then $F$ is equal to $\mathbb{Q}(j, j(N z))$ where $j$ is the modular invariant of $\Gamma(1)$.

Proof. Let $X\left(\Gamma^{\prime}\right)=X_{0}(N)$. We recall that $K\left(X_{0}(N)\right)=\mathbb{C}(j, j(N z))$ ([19], Proposition 2.10) and consider the field tower

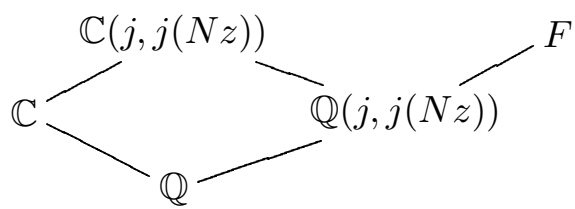

Since $F$ and $\mathbb{C}$ are linearly disjoint over $\mathbb{Q}$, we claim that $F$ and $\mathbb{C}(j, j(N z))$ are linearly disjoint over $\mathbb{Q}(j, j(N z))$. Therefore

$$
1 \leq[F: \mathbb{Q}(j, j(N z))] \leq[\mathbb{C} X: \mathbb{C}(j, j(N z))] \leq\left[K\left(X_{\Gamma^{\prime}}\right): K\left(X_{\Gamma^{\prime}}\right)\right]=1 .
$$

Consider the case $N=4$. Since $j_{1,4}$ has rational Fourier coefficients, from Lemmas 4 and 5 we derive

Theorem 6. $\mathbb{Q}(j, j(4 z))=\mathbb{Q}\left(j_{1,4}\right)$ is the field of all modular functions in $K\left(X_{1}(4)\right)$ whose Fourier coefficients with respect to $q$ are rational numbers.

Define $j_{4}(z)=\theta_{3}(z / 2) / \theta_{4}(z / 2)$. Let $F_{4}$ be the field of all modular functions of level 4 whose Fourier expansions with respect to $q_{4}$ have rational coefficients. Then by [19], Proposition 6.9 , we know that $F_{4}=\mathbb{Q}(j(z), j(4 z)$, $\left.f_{1,0}(z)\right)$ where $f_{1,0}(z)$ is a Fricke function. Also by [8], Theorem 18, we see that $F_{4}=\mathbb{Q}\left(j_{4}\right)$. Since $j_{1,4}$ has rational Fourier coefficients, we have 
$j_{1,4} \in F_{4}$. Hence we are able to express $j_{1,4}$ as a rational function of $j_{4}$. On the other hand, it is not difficult to derive that

$$
\theta_{2}(2 z)=\frac{1}{2}\left(\theta_{3}(z / 2)-\theta_{4}(z / 2)\right) \quad \text { and } \quad \theta_{3}(2 z)=\frac{1}{2}\left(\theta_{3}(z / 2)+\theta_{4}(z / 2)\right) \text {. }
$$

From the above we get

THEOREM 7.

$$
j_{1,4}(z)=\left(\frac{j_{4}(z)-1}{j_{4}(z)+1}\right)^{4} .
$$

4. Some remarks on Thompson series. Let $\Gamma$ be a Fuchsian group of the first kind and $f \in K(X(\Gamma))$. We call $f$ normalized if its $q$ series is $q^{-1}+0+a_{1} q+a_{2} q^{2}+\ldots$

LEMMA 8. The normalized generator of a genus zero function field is unique.

Proof. Let $\Gamma$ be a Fuchsian group such that the genus of the curve $\Gamma \backslash \mathfrak{H}^{*}$ is zero. Assume that $K(X(\Gamma))=\mathbb{C}\left(J_{1}\right)=\mathbb{C}\left(J_{2}\right)$ where $J_{1}$ and $J_{2}$ are normalized. We can then write their Fourier expansions as

$$
J_{1}=q^{-1}+0+a_{1} q+a_{2} q^{2}+\ldots \quad \text { and } \quad J_{2}=q^{-1}+0+b_{1} q+b_{2} q^{2}+\ldots
$$

Observe that $1=\left[K(X(\Gamma)): \mathbb{C}\left(J_{i}\right)\right]=\nu_{0}\left(J_{i}\right)=\nu_{\infty}\left(J_{i}\right)$ for $i=1,2$. Hence, $J_{1}$ and $J_{2}$ have only one zero and one pole whose orders are simple. We see that the only poles of $J_{i}$ occur at $\infty$. Then, $J_{1}-J_{2}$ has no poles because the two series start with $q^{-1}$. So, it should be a constant. Since $J_{1}-J_{2}=\left(a_{1}-b_{1}\right) q+\ldots$, this constant must be zero. This proves the lemma.

Let $\mathfrak{F}$ be the set of functions $f(z)$ satisfying the following conditions:

(i) $f(z) \in K\left(X\left(\Gamma^{\prime}\right)\right)$ for some discrete subgroup $\Gamma^{\prime}$ of $S L_{2}(\mathbb{R})$ that contains $\Gamma_{0}(N)$ for some $N$.

(ii) The genus of the curve $X\left(\Gamma^{\prime}\right)$ is 0 and its function field $K\left(X\left(\Gamma^{\prime}\right)\right)$ is equal to $\mathbb{C}(f)$.

(iii) In a neighborhood of $\infty, f(z)$ is expressed in the form:

$$
f(z)=q^{-1}+\sum_{n=0}^{\infty} a_{n} q^{n}, \quad a_{n} \in \mathbb{C} .
$$

We say that a pair $(G, \phi)$ is a moonshine for a finite group $G$ if $\phi$ is a function from $G$ to $\mathfrak{F}$ and the mapping $\sigma \rightarrow a_{n}(\sigma)$ from $G$ to $\mathbb{C}$ is a generalized character of $G$ when $\phi_{\sigma}(z)=q^{-1}+a_{0}(\sigma)+\sum_{n=1}^{\infty} a_{n}(\sigma) q^{n}$ for $\sigma \in G$. In particular, $\phi_{\sigma}$ is a class function of $G$.

Finding or constructing a moonshine $(G, \phi)$ for a given group $G$, however, involves some nontrivial work. This is because for each element $\sigma$ of $G$, we have to find a natural number $N$ and a Fuchsian group $\Gamma^{\prime}$ containing 
$\Gamma_{0}(N)$ in such a way that the function field $K\left(X\left(\Gamma^{\prime}\right)\right)$ is equal to $\mathbb{C}\left(\phi_{\sigma}\right)$ and the coefficients $a_{n}(\sigma)$ of the expansion of $\phi_{\sigma}(z)$ at $\infty$ induce generalized characters for all $n \geq 1$.

Let $j$ be the modular invariant of $\Gamma(1)$ whose $q$-series is

$$
j=q^{-1}+744+196884 q+\ldots=\sum_{r} c_{r} q^{r} .
$$

Then $j-744$ is the normalized generator of $\Gamma(1)$. Let $M$ be the monster simple group of order approximately $8 \cdot 10^{53}$. Thompson proposed that the coefficients in the $q$-series for $j-744$ be replaced by the representations of $M$ so that we obtain a formal series

$$
H_{-1} q^{-1}+0+H_{1} q+H_{2} q^{2}+\ldots
$$

in which the $H_{r}$ are certain representations of $M$ called head representations. $H_{r}$ has degree $c_{r}$ as in (9), for example, $H_{-1}$ is the trivial representation (degree 1 ), while $H_{1}$ is the sum of this and the degree 196883 representation and $\mathrm{H}_{2}$ is the sum of the former two and the degree 21296876 representation ([20]). The following theorem conjectured by Thompson and proved by Borcherds shows that there exists a moonshine for the monster group $M$.

THEOREM 9. The series

$$
T_{m}=q^{-1}+0+H_{1}(m) q+H_{2}(m) q^{2}+\ldots
$$

is the normalized generator of a genus zero function field arising from a group between $\Gamma_{0}(N)$ and its normalizer in $P S L_{2}(\mathbb{R})$, where $m$ is an element of $M$ and $H_{r}(m)$ is the character value of the head representation $H_{r}$ at $m$ $([1],[3])$.

Now we consider the case $\Gamma^{\prime}=\Gamma_{1}(4)$. We will then construct the normalized generator from the modular function $j_{1,4}$ mentioned in Theorem 3 . We have

$$
\begin{aligned}
\frac{16}{j_{1,4}(z)} & =\frac{16 \theta_{3}(2 z)^{4}}{\theta_{2}(2 z)^{4}} \\
& =\frac{\left(1+2 q+2 q^{4}+2 q^{9}+2 q^{16}+\ldots\right)^{4}}{q\left(1+q^{2}+q^{6}+q^{12}+q^{20}+\ldots\right)^{4}} \\
& =q^{-1}+8+20 q-62 q^{3}+216 q^{5}-641 q^{7}+1636 q^{9}+\ldots,
\end{aligned}
$$

which is in $q^{-1} \mathbb{Z}[[q]]$ because $q\left(1+q^{2}+q^{6}+q^{12}+\ldots\right)^{4} \in q \mathbb{Z}[[q]]^{\times}$. Let $N\left(j_{1,4}\right)=16 / j_{1,4}-8$. Then by [3], Lemma 8, Table 4 , and checking the coefficients of $q^{i}, i \leq 5$ ([1]) we have

THEOREM 10. $N\left(j_{1,4}\right)$ is the normalized generator of $K\left(X_{1}(4)\right)$, which corresponds to the Thompson series of type $4 C$. 
REMARK 11. Let $V=\bigoplus_{n \in \mathbb{Z}} V_{n}$ be the infinite-dimensional graded representation of the monster simple group constructed by Frenkel et al. ([6], [7]). For each element $m$ of the monster, we write the Thompson series as

$$
T_{m}=\sum_{n \in \mathbb{Z}} \operatorname{Tr}\left(m \mid V_{n}\right) q^{n}
$$

where $\operatorname{Tr}\left(m \mid V_{n}\right)$ is the trace of $m$ on the vector space $V_{n}$ and $q$ is a formal variable which can usually be thought of as a complex number with $|q|<1$. Let $m$ be the conjugacy class of order 4 and type $C$ in Atlas notation [2], and set

$$
N\left(j_{1,4}\right)=q^{-1}+\sum_{n \geq 1} H_{n}(m) q^{n} .
$$

Since $N\left(j_{1,4}\right)$ is the Thompson series of $m$ by Theorem 10, the results of [1] show that the coefficients $H_{n}(m)$ are the traces $\operatorname{Tr}\left(m \mid V_{n}\right)$ and satisfy the relation

$$
\begin{aligned}
p^{-1} \exp \left(-\sum_{i>0} \sum_{\substack{k>0 \\
n \in \mathbb{Z}}} \operatorname{Tr}\left(m^{i} \mid V_{k n}\right) p^{k i} q^{n i} / i\right) & \\
& =\sum_{k \in \mathbb{Z}} \operatorname{Tr}\left(m \mid V_{k}\right) p^{k}-\sum_{n \in \mathbb{Z}} \operatorname{Tr}\left(m \mid V_{n}\right) q^{n}
\end{aligned}
$$

where $p$ is also a formal variable which can be thought of as a complex number with $|p|<1$. The above identities then imply that $N\left(j_{1,4}\right)$ is completely replicable, and lead us to the recursion formulas (9.1) in [1], which later in this section turn out to be the same as ours (18) provided we put $H_{n}\left(m^{2}\right)=H_{n}^{(2)}$ (the coefficient of $q^{n}$ of the 2-plicate of $\left.N\left(j_{1,4}\right)\right)$.

Observe that $\bar{\Gamma}_{1}(4)=\bar{\Gamma}_{0}(4)$ as transformation groups, but the algorithm presented here is different from Conway-Norton's.

Following Norton's idea ([15], also see [1], [3] and [11]), we will state some replication formulas on the coefficients of $N\left(j_{1,4}\right)$. Let $N$ be a positive integer and $S$ be a subset of Hall divisors of $N$. By $N+S$ we mean the subgroup of $P S L_{2}(\mathbb{R})$ generated by $\Gamma_{0}(N)$ and all Atkin-Lehner involutions $W_{Q, N}$ for $Q \in S$. We assume that the genus of the curve $X(N+S)$ is zero. Let $t=q^{-1}+\sum_{m \geq 1} H_{m} q^{m}$ be the normalized generator of the function field of $X(N+S)$ as a completely replicable function. Then for each $n \geq 1$, there exists a unique polynomial $X_{n}(t)$ in $t$ such that $X_{n}(t) \equiv n^{-1} q^{-n} \bmod$ $q \mathbb{C}[[q]]$. In particular, $X_{1}(t)=t$. Write $X_{n}(t)=n^{-1} q^{-n}+\sum_{m \geq 1} H_{m, n} q^{m}$. Let $p=e^{2 \pi i y}$ for $y \in \mathfrak{H}$ and $q=e^{2 \pi i z}$ as usual. We then understand $t(y)$ and $t(z)$ as $p^{-1}+\sum_{m \geq 1} H_{m} p^{m}$ and $q^{-1}+\sum_{m \geq 1} H_{m} q^{m}$, respectively. Observe that $X_{n}(t)$ can be viewed as the coefficient of $p^{n}$ in $\log p^{-1}-\log (t(y)-t(z))$ 
([15], p. 185). To this end it suffices to show that

$$
\left.\frac{1}{n !} \cdot \frac{\partial^{n}}{\partial p^{n}}(-\log p-\log (t(y)-t(z)))\right|_{p=0}
$$

is a polynomial in $t$, which is congruent to $n^{-1} q^{-n} \bmod q \mathbb{C}[[q]]$. Since

$$
\log p^{-1}-\sum_{n \geq 1} X_{n}(t) p^{n}=\log (t(y)-t(z))
$$

we get by taking exponential on both sides,

$$
p^{-1} \exp \left(-\sum_{n \geq 1} X_{n}(t) p^{n}\right)=t(y)-t(z) .
$$

If we compare the coefficients of the terms $p^{2}, p^{3}$ and $p^{4}$ in (10), we have

$$
\begin{gathered}
\frac{1}{2}\left(t^{2}-2 X_{2}(t)\right)=H_{1}, \\
-\frac{1}{6}\left(t^{3}-6 t \cdot X_{2}(t)+6 X_{3}(t)\right)=H_{2}, \\
\frac{1}{24}\left(t^{4}-12 t^{2} \cdot X_{2}(t)+12 X_{2}(t)^{2}+24 t \cdot X_{3}(t)-24 X_{4}(t)\right)=H_{3} .
\end{gathered}
$$

Let $t^{(2)}$ be the normalized generator of the function field of $X\left(N^{(2)}+S^{(2)}\right)$ and define $t^{\left(2^{l}\right)}$ to be $\left(t^{\left(2^{l-1}\right)}\right)^{(2)}$, where $N^{(2)}=N /(2, N)$ and $S^{(2)}$ is the set of all $Q$ in $S$ which divide $N^{(2)}$. Write $t^{(s)}=q^{-1}+\sum_{m \geq 1} H_{m}^{(s)} q^{m}$. Also define the operator $U_{n}$ such that for $f(z)=\sum_{l \in \mathbb{Z}} a_{l} q^{l}$,

$$
\left.f(z)\right|_{U_{n}}=n \sum_{l \in \mathbb{Z}} a_{n l} q^{l}
$$

Then Koike ([11]) proved the following formulas called 2-plication and 4plication, respectively:

$$
\begin{gathered}
X_{2}(t)=\frac{1}{2}\left(\left.t\right|_{U_{2}}+t^{(2)}(2 \tau)\right), \\
X_{4}(t)=\frac{1}{4}\left(\left.t\right|_{U_{4}}+\left.t^{(2)}\right|_{U_{2}}(2 \tau)+t^{(4)}(4 \tau)\right) .
\end{gathered}
$$

Then by (11) and (14), it follows that

$$
\frac{1}{2} t^{2}-\frac{1}{2}\left(\left.t\right|_{U_{2}}+t^{(2)}(2 \tau)\right)=H_{1}
$$

Also by (11)-(15) we get

$$
\frac{1}{4}\left(\left.t\right|_{U_{2}}\right)^{2}+\left.\frac{1}{2} t\right|_{U_{2}} \cdot t^{(2)}(2 \tau)-H_{2} t-\left.\frac{1}{4} t\right|_{U_{4}}=H_{3}+\frac{1}{2} H_{1}^{2}-\frac{1}{2} H_{1}^{(2)} .
$$

If we compare the coefficients of $q^{2 k}$ and $q^{2 k+1}(k \geq 1)$ of both sides in (16) and (17) and carry out some routine calculation, we find that the coefficients of $t$ and $t^{(2)}$ satisfy the following recursion formulas for $k \geq 1$ : 


$$
\begin{aligned}
H_{4 k}= & H_{2 k+1}+\frac{H_{k}^{2}-H_{k}^{(2)}}{2}+\sum_{1 \leq j<k} H_{j} H_{2 k-j}, \\
H_{4 k+1}= & H_{2 k+3}-H_{2} H_{2 k}+\frac{H_{2 k}^{2}+H_{2 k}^{(2)}}{2}+\frac{H_{k+1}^{2}-H_{k+1}^{(2)}}{2} \\
& +\sum_{1 \leq j \leq k} H_{j} H_{2 k-j+2} \\
& +\sum_{1 \leq j<k} H_{j}^{(2)} H_{4 k-4 j}+\sum_{1 \leq j<2 k}(-1)^{j} H_{j} H_{4 k-j}, \\
H_{4 k+2}= & H_{2 k+2}+\sum_{1 \leq j \leq k} H_{j} H_{2 k-j+1}, \\
H_{4 k+3}= & H_{2 k+4}-H_{2} H_{2 k+1}-\frac{H_{2 k+1}^{2}-H_{2 k+1}^{(2)}}{2} \\
& +\sum_{1 \leq j \leq k+1} H_{j} H_{2 k-j+3} \\
& +\sum_{1 \leq j \leq k} H_{j}^{(2)} H_{4 k-4 j+2}+\sum_{1 \leq j \leq 2 k}(-1)^{j} H_{j} H_{4 k-j+2 .} .
\end{aligned}
$$

From the above formulas, we see that if $m=4$ or $m>5$ then $H_{m}$ is determined by the coefficients $H_{i}$ and $H_{i}^{(2)}$ for $1 \leq i<m$, so if we know all the coefficients $H_{m}^{(s)}$ for $m=1,2,3$, and 5 together with $s=2^{l}$ then we can work out all the coefficients $H_{m}$.

Now we take $N=4$ and $S=\{1\}$. Then $t$ is precisely $N\left(j_{1,4}\right)$ and $t^{(2)}$ is the normalized generator of the function field of $X_{0}(2)$ and for $l \geq 2$, $t^{\left(2^{l}\right)}$ is the normalized generator of the function field of $X_{0}(1)$. Hence we summarize the above results as follows.

THEOREM 12. If we know the 12 coefficients $H_{1}, H_{2}, H_{3}, H_{5}, H_{1}^{(2)}, H_{2}^{(2)}$, $H_{3}^{(2)}, H_{5}^{(2)}, H_{1}^{(4)}, H_{2}^{(4)}, H_{3}^{(4)}$ and $H_{5}^{(4)}$, then all the coefficients $H_{m}$ of the modular function $N\left(j_{1,4}\right)$ can be determined.

Observe that actually we do know the above 12 coefficients:

$$
\begin{aligned}
& H_{1}=20, H_{2}=0, H_{3}=-62, H_{5}=216 \text { by the definition of } N\left(j_{1,4}\right), \\
& H_{1}^{(2)}=276, H_{2}^{(2)}=-2048, H_{3}^{(2)}=11202, H_{5}^{(2)}=184024 \text { by }[10], \\
& H_{1}^{(4)}=196884, H_{2}^{(4)}=21493760, H_{3}^{(4)}=864299970, \\
& H_{5}^{(4)}=333202640600 \quad \text { by }[3] .
\end{aligned}
$$

Here, the modular function $j_{1,2}$ is defined by $j_{1,2}(z)=\theta_{2}(z)^{8} / \theta_{4}(2 z)^{8}$ for $z \in \mathfrak{H}$. 
It is worth finding when the modular function $N\left(j_{1,4}\right)$ could be an algebraic integer. We close this section by showing the following number theoretic result.

TheOREm 13. Let $d$ be a square-free positive integer. For $\tau \in \mathbb{Q}(\sqrt{-d}) \cap$ $\mathfrak{H}, N\left(j_{1,4}\right)(\tau)$ is an algebraic integer.

Proof. Let $j(z)=q^{-1}+744+196884 q+\ldots$ It is well known that $j(\tau)$ is an algebraic integer for $\tau \in \mathbb{Q}(\sqrt{-d}) \cap \mathfrak{H}$ ([13], [19]). For algebraic proofs, see [4], [14] and [18]. Let $J=j / 1728$. Then we know that

$$
J=\frac{4}{27} \cdot \frac{\left(\lambda^{2}-\lambda+1\right)^{3}}{\lambda^{2}(\lambda-1)^{2}} \quad \text { where } \quad \lambda=\frac{\theta_{2}(z)^{4}}{\theta_{3}(z)^{4}}=j_{1,4}\left(\frac{z}{2}\right)
$$

([16], p. 228). Hence,

$$
j(2 \tau)=2^{8} \cdot \frac{\left(j_{1,4}(\tau)^{2}-j_{1,4}(\tau)+1\right)^{3}}{j_{1,4}(\tau)^{2}\left(j_{1,4}(\tau)-1\right)^{2}}=\frac{\left(N^{2}-32 N+448\right)^{3}}{(N-24)^{2}(N-8)^{2}}
$$

where $N=N\left(j_{1,4}\right)(\tau)$. This implies that $N\left(j_{1,4}\right)(\tau)$ is integral over $\mathbb{Z}[j(2 \tau)]$. Therefore it is integral over $\mathbb{Z}$ for $\tau \in \mathbb{Q}(\sqrt{-d}) \cap \mathfrak{H}$.

5. Explicit class fields generated by the modular function $j_{1,4}$. Let $\Gamma$ be a Fuchsian group of the first kind. Then $\Gamma \backslash \mathfrak{H}^{*}(=X(\Gamma))$ is a compact Riemann surface. Hence, there exists a projective nonsingular algebraic curve $V$, defined over $\mathbb{C}$, biregularly isomorphic to $\Gamma \backslash \mathfrak{H}^{*}$. We specify a $\Gamma$-invariant holomorphic map $\varphi$ of $\mathfrak{H}^{*}$ to $V$ which gives a biregular isomorphism of $\Gamma \backslash \mathfrak{H}^{*}$ to $V$. In that situation, we call $(V, \varphi)$ a model of $\Gamma \backslash \mathfrak{H}^{*}$. Now we assume that the genus of $\Gamma \backslash \mathfrak{H}^{*}$ is zero. Then its function field $K(X(\Gamma))$ is equal to $\mathbb{C}\left(J^{\prime}\right)$ for some $J^{\prime} \in K(X(\Gamma))$.

Lemma 14. $\left(\mathbb{P}^{1}(\mathbb{C}), J^{\prime}\right)$ is a model of $\Gamma \backslash \mathfrak{H}^{*}$.

Proof. First, we view $J^{\prime}$ as a meromorphic function on $\Gamma \backslash \mathfrak{H}^{*}$. By defining

$$
J^{\prime}(z)= \begin{cases}{[1: 0]} & \text { if } z \text { is a pole } \\ {\left[J^{\prime}(z): 1\right]} & \text { otherwise }\end{cases}
$$

we get a holomorphic function of $\Gamma \backslash \mathfrak{H}^{*}$ to $\mathbb{P}^{1}$, as a map between compact Riemann surfaces. We denote it again by $J^{\prime}$. Now for any $c_{0} \in \mathbb{C}$, we consider $J^{\prime}-c_{0}$. Since $K(X(\Gamma))=\mathbb{C}\left(J^{\prime}\right)=\mathbb{C}\left(J^{\prime}-c_{0}\right)$ and $[K(X(\Gamma))$ : $\left.\mathbb{C}\left(J^{\prime}-c_{0}\right)\right]=\nu_{0}\left(J^{\prime}-c_{0}\right)$ where $\nu_{0}$ is the sum of orders of zeros, we have $\nu_{0}\left(J^{\prime}-c_{0}\right)=1$. Therefore there exists a unique point $z_{0} \in \Gamma \backslash \mathfrak{H}^{*}$ such that $J^{\prime}\left(z_{0}\right)=c_{0}$. This implies the bijectivity of $J^{\prime}$. Since any injective holomorphic mapping between two Riemann surfaces is biholomorphic ([5], Corollary 2.5), the assertion follows. 
Let $G_{\mathbb{A}}$ be the adelization of $G=G L_{2}(\mathbb{Q})$. Put

$$
\begin{aligned}
G_{p} & =G L_{2}\left(\mathbb{Q}_{p}\right) \quad(p \text { a rational prime }), \\
G_{\infty} & =G L_{2}(\mathbb{R}), \\
G_{\infty+} & =\left\{x \in G_{\infty} \mid \operatorname{det}(x)>0\right\}, \\
G_{\mathbb{Q}_{+}} & =\left\{x \in G L_{2}(\mathbb{Q}) \mid \operatorname{det}(x)>0\right\} .
\end{aligned}
$$

We define the topology of $G_{\mathbb{A}}$ by taking $U=\prod_{p} G L_{2}\left(\mathbb{Z}_{p}\right) \times G_{\infty+}$ to be an open subgroup of $G_{\mathbb{A}}$. Let $K$ be an imaginary quadratic field and $\xi$ be an embedding of $K$ into $M_{2}(\mathbb{Q})$. We call $\xi$ normalized if it is defined by $a\left(\begin{array}{c}z \\ 1\end{array}\right)=\xi(a)\left(\begin{array}{c}z \\ 1\end{array}\right)$ for $a \in K$ where $z$ is the fixed point of $\xi\left(K^{\times}\right)\left(\subset G_{\mathbb{Q}_{+}}\right)$in $\mathfrak{H}$. Observe that the embedding $\xi$ defines a continuous homomorphism of $K_{\mathbb{A}}^{\times}$into $G_{\mathbb{A}+}$, which we denote again by $\xi$. Here $G_{\mathbb{A}+}$ is the group $G_{0} G_{\infty+}$ with $G_{0}$ the nonarchimedean part of $G_{\mathbb{A}}$, and $K_{\mathbb{A}}^{\times}$is the idele group of $K$.

Let $\mathcal{Z}$ be the set of open subgroups $S$ of $G_{\mathbb{A}+}$ containing $\mathbb{Q}^{\times} G_{\infty+}$ such that $S / \mathbb{Q}^{\times} G_{\infty+}$ is compact. For $S \in \mathcal{Z}$, we see that $\operatorname{det}(S)$ is open in $\mathbb{Q}_{\mathbb{A}}^{\times}$. Therefore the subgroup $\mathbb{Q}^{\times} \cdot \operatorname{det}(S)$ of $\mathbb{Q}_{\mathbb{A}}^{\times}$corresponds to a finite abelian extension of $\mathbb{Q}$, which we write $k_{S}$. Put $\Gamma_{S}=S \cap G_{\mathbb{Q}_{+}}$for $S \in \mathcal{Z}$. Then it is known ([19], Proposition 6.27) that $\Gamma_{S} / \mathbb{Q}^{\times}$is a Fuchsian group of the first kind commensurable with $\Gamma(1) /\{ \pm 1\}$. Let $U^{\prime}=\left\{x=\left(x_{p}\right) \in U \mid x_{p} \in U_{p}^{\prime}\right.$ for all finite $p\}$ where $U_{p}^{\prime}=\left\{\left(\begin{array}{ll}a & b \\ c & d\end{array}\right) \in G L_{2}\left(\mathbb{Z}_{p}\right) \mid c \equiv 0 \bmod N \mathbb{Z}_{p}\right\}$. We then have

Lemma 15. (i) $\mathbb{Q}^{\times} U^{\prime} \in \mathcal{Z}$.

(ii) $k_{S}=\mathbb{Q}$, if $S=\mathbb{Q}^{\times} U^{\prime}$.

(iii) $\Gamma_{S}=\mathbb{Q}^{\times} \Gamma_{0}(N)$ if $S=\mathbb{Q}^{\times} U^{\prime}$.

Proof. First, we observe that $\mathbb{Q}^{\times} U^{\prime}$ is an open subgroup of $\mathbb{Q}^{\times} U$. Hence, for (i), it is enough to show that $\mathbb{Q}^{\times} U / \mathbb{Q}^{\times} G_{\infty+}$ is compact. But we know that $\mathbb{Q}^{\times} U / \mathbb{Q}^{\times} G_{\infty+}=\prod G L_{2}\left(\mathbb{Z}_{p}\right)$ is compact. For (ii), note that $\mathbb{Q}$ corresponds to the norm group $\mathbb{Q}^{\times} \cdot \mathbb{Q}_{\mathbb{A}}^{\times \infty}$ with $\mathbb{Q}_{\mathbb{A}}^{\times \infty}=\mathbb{R}^{\times} \times \prod_{p} \mathbb{Z}_{p}^{\times}$. We claim that $\operatorname{det} U^{\prime}=\mathbb{Q}_{\mathbb{A}}^{\times \infty}$. Indeed, it is obvious that $\operatorname{det} U^{\prime} \subset \mathbb{Q}_{\mathbb{A}}^{\times \infty}$. Conversely, for any element $\left(\alpha_{p}\right) \in \mathbb{Q}_{\mathbb{A}}^{\times \infty}$, take $y_{p}=\left(\begin{array}{cc}1 & 0 \\ 0 & \alpha_{p}\end{array}\right)$. Then $\left(y_{p}\right) \in U^{\prime}$ and $\operatorname{det}\left(y_{p}\right)=\left(\operatorname{det} y_{p}\right)=\left(\alpha_{p}\right)$. Finally, if $S=\mathbb{Q}^{\times} U^{\prime}$ then we come up with $\Gamma_{S}=\mathbb{Q}^{\times} U^{\prime} \cap G_{\mathbb{Q}_{+}}=\mathbb{Q}^{\times}\left(U^{\prime} \cap G_{\mathbb{Q}_{+}}\right)=\mathbb{Q}^{\times} \Gamma_{0}(N)$

REMARK 16. For $z \in K \cap \mathfrak{H}$, we consider a normalized embedding $\xi_{z}$ : $K \rightarrow M_{2}(\mathbb{Q})$ defined by $a\left(\begin{array}{l}z \\ 1\end{array}\right)=\xi_{z}(a)\left(\begin{array}{c}z \\ 1\end{array}\right)$ for $a \in K$. Then $z$ is the fixed point of $\xi_{z}\left(K^{\times}\right)$in $\mathfrak{H}$. Let $\left(V_{S}, \varphi_{S}\right)$ be a model of $\Gamma_{S} \backslash \mathfrak{H}^{*}$. By Lemma 15(iii), $\Gamma_{S}=\mathbb{Q}^{\times} \Gamma_{0}(4)=\mathbb{Q}^{\times} \Gamma_{1}(4)$ when $S=\mathbb{Q}^{\times} U^{\prime}$ with $N=4$. By Theorem 3 and Lemma 14, we can take $\varphi_{S}=j_{1,4}$ and $V_{S}=\mathbb{P}^{1}$. Now it follows from [19], Proposition 6.31 (ii), that $j_{1,4}(z)$ belongs to $\mathbb{P}^{1}\left(K^{\mathrm{ab}}\right)$ where $K^{\text {ab }}$ is the maximal abelian extension of $K$. Furthermore, $\theta_{i}(z)$ has no zeros in $\mathfrak{H}$ for $i=2,3,4$. Hence, $j_{1,4}(z)$ in fact is in $K^{\mathrm{ab}}$ for $z \in K \cap \mathfrak{H}$. 
TheOREM 17. Let $K$ be an imaginary quadratic field and let $\xi_{z}$ be the normalized embedding for $z \in K \cap \mathfrak{H}$. Then $j_{1,4}(z) \in K^{\mathrm{ab}}$ and $K\left(j_{1,4}(z)\right)$ is a class field of $K$ corresponding to the subgroup $K^{\times} \cdot \xi_{z}^{-1}\left(\mathbb{Q}^{\times} U^{\prime}\right)$ of $K_{\mathbb{A}}^{\times}$.

Proof. From Lemma 15(ii) and (iii), if $S=\mathbb{Q}^{\times} U^{\prime}$ with $N=4$ then $k_{S}=\mathbb{Q}$ and $\Gamma_{S}=\mathbb{Q}^{\times} \Gamma_{1}(4)$. Since $j_{1,4}$ gives a model of the curve $X_{1}(4)$, we can take $\varphi_{S}=j_{1,4}$. Now the assertion follows from [19], Proposition 6.33 and Remark 16.

In view of standard results on complex multiplication, it is interesting to investigate whether the value $N\left(j_{1,4}\right)(\alpha)$ is a generator for a certain full ray class field if $\alpha$ is the quotient of a basis of an ideal belonging to the maximal order in $\mathbb{Q}(\sqrt{-d})$. We first need a result on complex multiplication.

THEOREM 18. Let $\mathfrak{F}_{N}$ be the field of modular functions of level $N$ rational over $\mathbb{Q}\left(e^{2 \pi i / N}\right)$, and let $k$ be an imaginary quadratic field. Let $\mathfrak{O}_{k}$ be the maximal order of $k$ and $\mathfrak{A}$ be an $\mathfrak{O}_{k}$-ideal such that $\mathfrak{A}=\left[z_{1}, z_{2}\right]$ and $z=$ $z_{1} / z_{2} \in \mathfrak{H}$. Then the field $k \mathfrak{F}_{N}(z)$ generated over $k$ by all values $f(z)$ with $f \in \mathfrak{F}_{N}$ and $f$ defined at $z$ is the ray class field over $k$ with conductor $N$.

P r o of. [13], Ch. 10, Corollary of Theorem 2.

REMARK 19. When $N=2, \mathfrak{F}_{2}$ is the field of all modular functions of level 2 rational over $\mathbb{Q}$. On the other hand, it is a well-known fact that $K(X(\Gamma(2)))=\mathbb{C}(\lambda)$ where $\lambda$ is the classical modular function of level 2 . Then by Lemma $4, \mathfrak{F}_{2}=\mathbb{Q}(\lambda)$. Hence by Theorem $18, k(\lambda(z))$ is the ray class field over $k$ with conductor 2 where $z$ is chosen as in the theorem.

TheOREm 20. Let $k$ and $\mathfrak{O}_{k}$ be as in Theorem 18. Put $\mathfrak{O}_{k}=x \mathbb{Z}+\mathbb{Z}$ and $\mathfrak{A}=x \mathbb{Z}+2 \mathbb{Z}$ for $x \in \mathfrak{H}$. If $N_{k / \mathbb{Q}}(x)$ is an even integer, then $\mathfrak{A}$ is an $\mathfrak{O}_{k}$-ideal and $N\left(j_{1,4}\right)(x / 2)$ generates a ray class field over $k$ with conductor 2 .

P r o of. Note that $\mathfrak{A}$ is an $\mathfrak{O}_{k}$-ideal if and only if $x \cdot \mathfrak{A} \subseteq \mathfrak{A}$. Since $x \cdot \mathfrak{A}=x^{2} \mathbb{Z}+2 x \mathbb{Z}, x \cdot \mathfrak{A} \subseteq \mathfrak{A}$ is equivalent to $x^{2} \in \mathfrak{A}$. Let $x^{2}-\operatorname{Tr}_{k / \mathbb{Q}}(x) \cdot x+$ $N_{k / \mathbb{Q}}(x)=0$ be the equation of $x$. Since $\operatorname{Tr}_{k / \mathbb{Q}}(x)$ and $N_{k / \mathbb{Q}}(x)$ are in $\mathbb{Z}$, we have $x^{2} \in \mathfrak{A}$ if and only if $N_{k / \mathbb{Q}}(x) \in 2 \mathbb{Z}$. Next, we observe that

Hence

$$
\lambda(z)=\frac{\theta_{2}(z)^{4}}{\theta_{3}(z)^{4}}=j_{1,4}\left(\frac{z}{2}\right) \quad \text { and } \quad N\left(j_{1,4}\right)=\frac{16}{j_{1,4}}-8 .
$$

$$
k\left(N\left(j_{1,4}\right)\left(\frac{x}{2}\right)\right)=k\left(j_{1,4}\left(\frac{x}{2}\right)\right)=k(\lambda(x))
$$

is the ray class field with conductor 2 by Remark 19 .

Corollary 21. With the notations of Theorem $20, N\left(j_{1,4}\right)(x / 2)$ belongs to the maximal order in the ray class field $k(\lambda(x))$ over $k$ with conductor 2 .

Proof. This is immediate from Theorems 13 and 20. 


\section{References}

[1] R. E. Borcherds, Monstrous moonshine and monstrous Lie superalgebras, Invent. Math. 109 (1992), 405-444.

[2] J. H. Conway, R. T. Curtis, S. P. Norton, R. A. Parker, and R. A. Wilson, Atlas of Finite Groups, Clarendon Press, 1985.

[3] J. H. Conway and S. P. Norton, Monstrous moonshine, Bull. London Math. Soc. 11 (1979), 308-339.

[4] M. Deuring, Die Typen der Multiplikatorenringe elliptischer Funktionenkörper, Abh. Math. Sem. Univ. Hamburg 14 (1941), 197-272.

[5] O. Foster, Lectures on Riemann Surfaces, Springer, 1981.

[6] I. B. Frenkel, J. Lepowsky, and A. Meurman, Vertex Operator Algebras and the Monster, Academic Press, Boston, 1988.

[7] - - - - - A natural representation of the Fischer-Griess monster with the modular function $J$ as character, Proc. Nat. Acad. Sci. U.S.A. 81 (1984), 3256-3260.

[8] C. H. Kim and J. K. Koo, On the modular function $j_{4}$ of level 4, preprint.

[9] - - - On the genus of some modular curve of level N, Bull. Austral. Math. Soc. 54 (1996), 291-297.

[10] - - - On the modular function $j_{1,2}$, in preparation.

[11] M. Koike, On replication formula and Hecke operators, preprint, Nagoya University.

[12] S. Lang, Algebra, Addison-Wesley, 1993.

[13] —, Elliptic Functions, Springer, 1987.

[14] A. Néron, Modèles minimaux des variétés abéliennes sur les corps locaux et globaux, Publ. Math. I.H.E.S. 21 (1964), 5-128.

[15] S. P. Norton, More on moonshine, in: Computational Group Theory, Academic Press, London, 1984, 185-195.

[16] R. Rankin, Modular Forms and Functions, Cambridge Univ. Press, Cambridge, 1977.

[17] B. Schoeneberg, Elliptic Modular Functions, Springer, 1973.

[18] J.-P. Serre and J. Tate, Good reduction of abelian varieties, Ann. of Math. 88 (1968), 492-517.

[19] G. Shimura, Introduction to the Arithmetic Theory of Automorphic Functions, Publ. Math. Soc. Japan 11, Tokyo, 1971.

[20] J. G. Thompson, Some numerology between the Fischer-Griess monster and the elliptic modular function, Bull. London Math. Soc. 11 (1979), 352-353.

Department of Mathematics

Korea Advanced Institute of Science and Technology

Taejon 305-701, Korea

E-mail: kch@math.kaist.ac.kr

jkkoo@math.kaist.ac.kr

Received on 6.12.1996

and in revised form on 1.4.1997 